\title{
GENERALIZATIONS OF PRINCIPALLY QUASI-INJECTIVE MODULES AND QUASIPRINCIPALLY INJECTIVE MODULES
}

\author{
ZHU ZHANMIN, XIA ZHANGSHENG, AND TAN ZHISONG
}

Received 22 November 2004 and in revised form 9 June 2005

Let $R$ be a ring and $M$ a right $R$-module with $S=\operatorname{End}\left(M_{R}\right)$. The module $M$ is called almost principally quasi-injective (or APQ-injective for short) if, for any $m \in M$, there exists an $S$-submodule $X_{m}$ of $M$ such that $l_{M} r_{R}(m)=S m \oplus X_{m}$. The module $M$ is called almost quasiprincipally injective (or AQP-injective for short) if, for any $s \in S$, there exists a left ideal $X_{s}$ of $S$ such that $l_{S}(\operatorname{ker}(s))=S s \oplus X_{s}$. In this paper, we give some characterizations and properties of the two classes of modules. Some results on principally quasi-injective modules and quasiprincipally injective modules are extended to these modules, respectively. Specially in the case $R_{R}$, we obtain some results on AP-injective rings as corollaries.

\section{Introduction}

Throughout $R$ is a ring with identity and $M$ is a right $R$-module with $S=\operatorname{End}\left(M_{R}\right)$. Recall a ring $R$ is called right principally injective [5] (or right P-injective for short) if, every homomorphism from a principally right ideal of $R$ to $R$ can be extended to an endomorphism of $R$, or equivalently, $\operatorname{lr}(a)=R a$ for all $a \in R$. The notion of right P-injective rings has been generalized by many authors. For example, in $[4,8]$, right P-injective rings are generalized to modules in two ways, respectively. Following [4], the module $M$ is called principally quasi-injective (or PQ-injective for short) if, each $R$-homomorphism from a principal submodule of $M$ to $M$ can be extended to an endomorphism of $M$. This is equivalent to saying that $l_{M} r_{R}(m)=S m$ for all $m \in M$, where $l_{M} r_{R}(m)$ consists of all elements $z \in M$ such that $m x=0$ implies $z x=0$ for any $x \in R$. In [8], the module $M$ is called quasiprincipally injective (or QP-injective for short) if, every homomorphism from an $M$-cyclic submodule of $M$ to $M$ can be extended to an endomorphism of $M$, or equivalently, $l_{S}(\operatorname{ker}(s))=S s$ for all $s \in S$. In [6], right P-injective rings are generalized to almost principally injective rings, that is, a ring $R$ is said to be almost principally injective (or AP-injective for short) if, for any $a \in R$, there exists a left ideal $X_{a}$ such that $\operatorname{lr}(a)=R a \oplus X_{a}$. The nice structure of PQ-injective modules, QP-injective modules, and $\mathrm{AP}$-injective rings draws our attention to define almost $\mathrm{PQ}$-injective modules and almost $\mathrm{QP}$-injective modules in similar ways to AP-injective rings, and to investigate their characterizations and properties. 


\section{Generalizations of principally quasi-injective modules}

\section{APQ-injective modules}

Definition 2.1. Let $M$ be a right $R$-module and let $S=\operatorname{End}\left(M_{R}\right)$. The module $M$ is called almost principally quasi-injective (briefly, APQ-injective) if, for any $m \in M$, there exists an $S$-submodule $X_{m}$ of $M$ such that $l_{M} r_{R}(m)=S m \oplus X_{m}$.

The concept of APQ-injective modules is explained by the following lemma.

LeMmA 2.2. Let $M_{R}$ be a module and let $S=\operatorname{End}\left(M_{R}\right)$, and $m \in M$.

(1) If $l_{M} r_{R}(m)=S m \bigoplus X$ for some $X \subseteq M$ as left $S$-modules, then $\operatorname{Hom}_{R}(m R, M)$ $=S \bigoplus \Gamma$ as left $S$-modules, where $\Gamma=\left\{f \in \operatorname{Hom}_{R}(m R, M) \mid f(m) \in X\right\}$.

(2) If $\operatorname{Hom}_{R}(m R, M)=S \oplus \Gamma$ as left S-modules, then $l_{M}\left(r_{R}(m)\right)=S m \oplus X$ as left $S$ modules, where $X=\{f(m) \mid f \in \Gamma\}$.

(3) Sm is a summand of $l_{M}\left(r_{R}(m)\right)$ as left $S$-modules if and only if $S$ is a summand of $\operatorname{Hom}_{R}(m R, M)$ as left $S$-modules.

Proof. The map $\theta: l_{M}\left(r_{R}(m)\right) \rightarrow \operatorname{Hom}_{R}(m R, M)$ with $\theta(a)=\lambda_{a}$ is a left $S$-isomorphism, where $\lambda_{a}: m R \rightarrow M$ is defined by $\lambda_{a}(m r)=a r$, so the lemma follows. Moreover, $s(S m)$ is nonsmall in $l_{M}\left(r_{R}(m)\right)$ if and only if $S$ is nonsmall in $\operatorname{Hom}_{R}(m R, M)$.

From Lemma 2.2, the following corollary follows.

Corollary 2.3 [4, Lemma 1.1]. Let $M_{R}$ be a right $R$-module with $S=\operatorname{End}\left(M_{R}\right)$ and $m \in$ $M$. Then $l_{M}\left(r_{R}(m)\right)=S m$ if and only if every $R$-homomorphism of $m R$ into $M$ extends to $M$.

From Corollary 2.3, we see that all PQ-injective modules are APQ-injective. Since a ring $R$ is right $\mathrm{P}$-injective (resp., AP-injective) if and only if the right $R$-module $R_{R}$ is PQ-injective (resp., APQ-injective), and Page and Zhou [6] have given three examples of rings which are right $\mathrm{AP}$-injective but not right $\mathrm{P}$-injective, so in general, $\mathrm{APQ}$-injective modules need not be PQ-injective.

Recall that a ring $R$ is called right QP-injective [6, Definition 2.1], if for any $0 \neq a \in R$, there exists a left ideal $X_{a}$ such that $\operatorname{lr}(a)=R a+X_{a}$ with $a \notin X_{a}$. Now we extend this concept to modules.

Definition 2.4. Let $M$ be a right $R$-module with $S=\operatorname{End}\left(M_{R}\right)$, the module $M$ is said to be QPQ-injective (i.e., quasiprincipally quasi-injective) if, for any nonzero element $m$ of $M$, there exists an $S$-submodule $X_{m}$ of $M$ such that $l_{M} r_{R}(m)=S m+X_{m}$ with $m \notin X_{m}$.

Clearly, right APQ-injective modules are QPQ-injective, but the reverse implication is not true. For example, $Z$-module $Z_{Z}$ is QPQ-injective, but not APQ-injective.

Let $M$ be a right $R$-module with $S=\operatorname{End}\left(M_{R}\right)$, and $J(S)$ the Jacobson radical of $S$. Following [4], write $W(S)=\left\{w \in S \mid \operatorname{ker}(w) \subseteq{ }^{\text {ess }} M\right\}$.

Theorem 2.5. Let $M_{R}$ be QPQ-injective with $S=\operatorname{End}\left(M_{R}\right)$. Then

(1) $J(S) \subseteq W(S)$,

(2) $\operatorname{Soc}\left(M_{R}\right) \subseteq r_{M}(J(S))$.

Proof. (1) Let $a \in J(S)$. If $a \notin W(S)$, then $\operatorname{ker}(a) \bigcap K=0$ for some $0 \neq K \leq M_{R}$. Take $k \in K$ such that $a k \neq 0$, then $l_{M}\left(r_{R}(a k)\right)=S(a k)+X_{a k}$ with $a k \notin X_{a k}$. If $r \in r_{R}(a k)$, then $k r \in \operatorname{ker}(a) \bigcap K$, so $k r=0$, and hence $r \in r_{R}(k)$. This shows that $r_{R}(a k)=r_{R}(k)$. Note that 
$k \in l_{M}\left(r_{R}(k)\right)=l_{M}\left(r_{R}(a k)\right)=S(a k)+X_{a k}$, so we may write $k=b(a k)+x$, where $b \in S$ and $x \in X$. Then $(1-b a) k=x$, and so $k=(1-b a)^{-1} x$. Thus $a k=a(1-b a)^{-1} x \in X_{a k}$, a contradiction.

(2) Let $m R \subseteq M$ be simple. Suppose $a m \neq 0$ for some $a \in J(S)$. Then, since $m R$ is simple, $r_{R}(a m)=r_{R}(m)$. Since $M_{R}$ is QPQ-injective, there is a left $S$-module $X$ such that $a m \notin X$ and $l_{M} r_{R}(a m)=S(a m)+X$. Note that $m \in l_{M} r_{R}(a m)$, and so we may write $m=$ $b(a m)+x$, where $b \in S$ and $x \in X$. Then $(1-b a) m=x$, so $m=(1-b a)^{-1} x \in X$. This means that $a m \in X$, a contradiction.

Corollary 2.6. Let $M_{R}$ be QPQ-injective with $S=\operatorname{End}\left(M_{R}\right)$. If $S$ is semilocal, then $\operatorname{Soc}\left(M_{R}\right) \subseteq \operatorname{Soc}\left({ }_{S} M\right)$.

Proof. This follows from Theorem 2.5(2) and [1, Proposition 15.17].

Lemma 2.7. Let $M_{R}$ be APQ-injective with $S=\operatorname{End}\left(M_{R}\right)$. If $s \notin W(S)$, then the inclusion $\operatorname{ker}(s) \subset \operatorname{ker}(s-s t s)$ is strict for some $t \in S$.

Proof. If $s \notin W(S)$, then $\operatorname{ker}(s) \bigcap m R=0$ for some $0 \neq m \in M$. Thus $r_{R}(m)=r_{R}(\mathrm{sm})$, and so $l_{M} r_{R}(m)=l_{M} r_{R}(s m)=S(s m) \oplus X_{s m}$ as left $S$-modules because $M_{R}$ is APQ-injective. Write $m=t(s m)+x$, where $x \in X_{s m}$. Then $(s-s t s) m=s x \in S(s m) \cap X_{s m}$, and hence $(s-$ sts) $m=0$. Therefore, the inclusion $\operatorname{ker}(s) \subset \operatorname{ker}(s-s t s)$ is strict.

Lemma 2.8. Let $M$ be a right $R$-module with $S=\operatorname{End}\left(M_{R}\right)$. Suppose that for any sequence $\left\{s_{1}, s_{2}, \ldots\right\} \subseteq S$, the chain $\operatorname{ker}\left(s_{1}\right) \subseteq \operatorname{ker}\left(s_{2} s_{1}\right) \subseteq \cdots$ terminates. Then

(1) $W(S)$ is right T-nilpotent,

(2) $S / W(S)$ contains no infinite set of nonzero pairwise orthogonal idempotents.

Proof. This is a corollary of [2, Lemma 1.9].

Theorem 2.9. Let $M_{R}$ be APQ-injective with $S=\operatorname{End}\left(M_{R}\right)$, then the following conditions are equivalent.

(1) $S$ is right perfect.

(2) For any sequence $\left\{s_{1}, s_{2}, \ldots\right\} \subseteq S$, the chain $\operatorname{ker}\left(s_{1}\right) \subseteq \operatorname{ker}\left(s_{2} s_{1}\right) \subseteq \cdots$ terminates.

Proof. (1) $\Rightarrow(2)$. Let $s_{i} \in S, i=1,2, \ldots$. Since $S$ is right perfect, $S$ satisfies DCC on principal left ideals. So the chain $S s_{1} \supseteq S s_{2} s_{1} \supseteq \cdots$ terminates. Thus there exists $n>0$ such that $S\left(s_{n} \cdots s_{1}\right)=S\left(s_{n+1} s_{n} \cdots s_{1}\right)=\cdots$. It follows that $\operatorname{ker}\left(s_{n} \cdots s_{1}\right)=\operatorname{ker}\left(s_{n+1} s_{n} \cdots s_{1}\right)=\cdots$.

$(2) \Rightarrow(1)$. First we prove that $S / W(S)$ is von Neumann regular. Let $s_{1} \notin W(S)$. Then $\operatorname{ker}\left(s_{1}\right)$ is not essential in $M$. By Lemma 2.7, there exists $t_{1} \in S$ such that $\operatorname{ker}\left(s_{1}\right) \subset \operatorname{ker}\left(s_{1}-\right.$ $\left.s_{1} t_{1} s_{1}\right)$ is proper. Put $s_{2}=s_{1}-s_{1} t_{1} s_{1}$. If $s_{2} \in W(S)$, then we have $\overline{s_{1}}=\overline{s_{1}} \cdot \overline{t_{1}} \cdot \overline{s_{1}}$ in the ring $S / W(S)$. If $s_{2} \notin W(S)$, then there exists $s_{3} \in S$ such that $\operatorname{ker}\left(s_{2}\right) \subset \operatorname{ker}\left(s_{3}\right)$ is proper, where $s_{3}=s_{2}-s_{2} t_{2} s_{2}$ for some $t_{2} \in S$ by the preceding proof. Repeating the above process, we get a strictly ascending chain

$$
\operatorname{ker}\left(s_{1}\right) \subset \operatorname{ker}\left(s_{2}\right) \subset \operatorname{ker}\left(s_{3}\right) \subset \cdots,
$$

where $s_{i+1}=s_{i}-s_{i} t_{i} s_{i}$ for some $t_{i} \in S, i=1,2, \ldots$. Let $u_{1}=s_{1}, u_{2}=1-s_{1} t_{1}, u_{3}=1-$ $s_{2} t_{2}, \ldots, u_{i+1}=1-s_{i} t_{i}, \ldots$ Then $s_{1}=u_{1}, s_{2}=u_{2} u_{1}, s_{3}=u_{3} u_{2} u_{1}, \ldots, s_{i+1}=u_{i+1} u_{i} \cdots u_{2} u_{1}, \ldots$, 
1856 Generalizations of principally quasi-injective modules

whence we have the following strict ascending chain

$$
\operatorname{ker}\left(u_{1}\right) \subset \operatorname{ker}\left(u_{2} u_{1}\right) \subset \operatorname{ker}\left(u_{3} u_{2} u_{1}\right) \subset \cdots,
$$

which contradicts the hypothesis. So there exists a positive integer $n$ such that $s_{n+1} \in$ $W(S)$. This shows that $\overline{s_{n}}$ is a regular element of $S / W(S)$, and hence $\overline{s_{n-1}}, \overline{s_{n-2}}, \ldots, \overline{s_{1}}$ are regular elements of $S / W(S)$. Thus $S / W(S)$ is regular.

Note that since $M_{R}$ is APQ-injective, $J(S) \subseteq W(S)$ by Theorem 2.5(1). Since the chain $\operatorname{ker}\left(s_{1}\right) \subseteq \operatorname{ker}\left(s_{2} s_{1}\right) \subseteq \cdots$ terminates, by Lemma $2.8(1), W(S)$ is right $T$-nilpotent, and so it follows that $W(S) \subseteq J(S)$, and thus $S / J(S)$ is regular. By Lemma 2.8, we get that $S$ is right perfect.

By Lemma 2.8 (1) and [7, Remark 2], we have the following lemma.

Lemma 2.10. Let $M$ be a right $R$-module with $S=\operatorname{End}\left(M_{R}\right)$. If $M_{R}$ satisfies $A C C$ on $\left\{r_{M}(A) \mid A \subseteq S\right\}$, then $W(S)$ is nilpotent.

The next corollary follows from Theorem 2.9 and Lemma 2.10.

Corollary 2.11. Let $M_{R}$ be APQ-injective with $S=\operatorname{End}\left(M_{R}\right)$. If $M_{R}$ satisfies ACC on $\left\{r_{M}(A) \mid A \subseteq S\right\}$, then $S$ is semiprimary.

For a module $M_{R}$, a submodule $X$ of $M$ is called a kernel submodule if $X=\operatorname{ker}(f)$ for some $f \in \operatorname{End}\left(M_{R}\right)$, and $X$ is called an annihilator submodule if $X=\bigcap_{f \in A} \operatorname{ker}(f)$ for some $A \subseteq \operatorname{End}\left(M_{R}\right)$.

Corollary 2.12. Let $M_{R}$ be an APQ-injective module and $S=\operatorname{End}\left(M_{R}\right)$. Then

(1) if $M_{R}$ satisfies ACC on kernel submodules, then $S$ is right perfect,

(2) if $M_{R}$ satisfies ACC on annihilator submodules, then $S$ is semiprimary.

\section{AQP-injective modules}

In this section we study a generalization of quasiprincipally injective modules.

Definition 3.1. Let $M$ be a right $R$-module with $S=\operatorname{End}\left(M_{R}\right)$. Then $M$ is said to be almost quasiprincipally injective (briefly, AQP-injective) if, for any $s \in S$, there exists a left ideal $X_{s}$ of $S$ such that $l_{S}(\operatorname{ker}(s))=S s \oplus X_{s}$ as left $S$-modules.

The next result gives the relationship between the AQP-injectivity of a module and the AP-injectivity of its endomorphism ring.

Theorem 3.2. Let $M_{R}$ be a right $R$-module with $S=\operatorname{End}\left(M_{R}\right)$. Then

(1) if $S$ is right AP-injective, then $M_{R}$ is AQP-injective,

(2) if $M_{R}$ is AQP-injective and $M$ generates $\operatorname{ker}(s)$ for each $s \in S$, then $S$ is right APinjective.

Proof. (1) Let $s \in S$. Since $S$ is right AP-injective, there exists a left ideal $I_{s}$ such that $l_{S} r_{S}(s)=S s \bigoplus I_{s}$. If $a \in l_{S}(\operatorname{ker}(s))$ and $b \in r_{S}(s)$, then $s b=0$, so $b M \subseteq \operatorname{ker}(s)$, and hence $a b M=0$, that is, $a b=0$. It follows that $l_{S}(\operatorname{ker}(s)) \subseteq l_{S} r_{S}(s)$. Thus, we have $S s \subseteq l_{S}(\operatorname{ker}(s)) \subseteq$ $S s \oplus I_{s}$. This shows that $l_{S}(\operatorname{ker}(s))=S s \bigoplus l_{S}(\operatorname{ker}(s)) \bigcap I_{s}$, and (1) is proved. 
(2) Let $0 \neq s \in S$. As $M_{R}$ is AQP-injective, $l_{S}(\operatorname{ker}(s))=S s \oplus X_{s}$ for some left ideal $X_{s}$ of $S$. Assume $a \in l_{S} r_{S}(s)$. Since $M$ generates $\operatorname{ker}(s), \operatorname{ker}(s)=\sum_{t \in T} t(M)$ for some subset $T$ of $S$. It is easy to see that $a t=0$ for each $t \in T$, thus $a x=0$ for each $x \in \operatorname{ker}(s)$. This implies that $l_{S} r_{S}(s) \subseteq l_{S}(\operatorname{ker}(s))$, from which we have $S s \subseteq l_{S} r_{S}(s) \subseteq S s \bigoplus X_{s}$, and hence $l_{S} r_{S}(s)=S s \bigoplus\left(l_{S} r_{S}(s) \cap X_{s}\right)$. Therefore, $S$ is right AP-injective.

Theorem 3.3. Let $M$ be a right $R$-module with $S=\operatorname{End}\left(M_{R}\right)$. If $M$ is an AQP-injective module which is a self-generator, then $J(S)=W(S)$.

Proof. Let $s \in J(S)$. Then we will show that $s \in W(S)$. If not, then there exists a nonzero submodule $K$ of $M$ such that $\operatorname{ker}(s) \cap K=0$. As $M$ is a self-generator, $K=\sum_{t \in I} t(M)$ for some subset $I$ of $S$, hence we have some $0 \neq t \in I$ such that $\operatorname{ker}(s) \cap t(M)=0$. Clearly, $s t \neq 0$ and $\operatorname{ker}(s t)=\operatorname{ker}(t)$. Since $M$ is AQP-injective, $l_{S}(\operatorname{ker}(s t))=S(s t) \oplus X_{s t}$ as left $S$ modules. Now $t \in l_{S}(\operatorname{ker}(t))=l_{S}(\operatorname{ker}(s t))=S(s t) \oplus X_{s t}$. Write $t=u(s t)+v$, where $u \in S$ and $v \in X_{s t}$. Then $s t-s u(s t)=s v \in S(s t) \bigcap X_{s t}$, hence $s t-s u(s t)=0$, that is, $(1-s u) s t=$ 0 . Note that $1-s u$ is left invertible, so $s t=0$, a contradiction.

Conversely, let $s \in W(S)$. Then, for each $t \in S, t s \in W(S)$ and so $1-t s \neq 0$. Since $M_{R}$ is AQP-injective, $l_{S}(\operatorname{ker}(1-t s))=S(1-t s) \bigoplus X_{1-t s}$ as left $S$-modules. Note that $\operatorname{ker}(t s) \cap$ $\operatorname{ker}(1-t s)=0$, so we have $\operatorname{ker}(1-t s)=0$, thus $S=S(1-t s) \bigoplus X_{1-t s}$, and then $1=e+x$ for some $e \in S(1-t s)$ and $x \in X$. It follows that $e^{2}=e$ and $S e=S(1-t s)$, and so $1-t s=$ $u$ for some $u \in S$. Since $\operatorname{ker}(t s)$ is essential in $M_{R}$, if $e \neq 1$, there is a nonzero element $(1-e) m \in(1-e) M \bigcap \operatorname{ker}(t s)$. Then $(1-t s)(1-e) m=(1-e) m$. But $(1-t s)(1-e) m=$ $u e(1-e) m=0$. This is a contradiction. So $e=1$ and hence $1-t s$ is left invertible. The result follows.

Recall that a module $M_{R}$ is said to satisfy the $C_{2}$-condition if every submodule of $M$ that is isomorphic to a direct summand of $M$ is itself a direct summand of $M$. A module $M$ is said to satisfy the $C_{3}$-condition if whenever $M_{1}$ and $M_{2}$ are two summands of $M$ and $M_{1} \cap M_{2}=0$, then $M_{1} \oplus M_{2}$ is a summand of $M$. It is well known that the $C_{2}$-condition implies the $C_{3}$-condition.

Theorem 3.4. If $M_{R}$ is an AQP-injective module, then it satisfies the $C_{2}$-condition. In particular, right $\mathrm{AP}$-injective rings are right $\mathrm{C}_{2}$-rings.

Proof. Let $A$ be a direct summand of $M$ with $A \cong B$ and $S=\operatorname{End}\left(M_{R}\right)$. Let $A=e M$, let $e^{2}=e \in S$, and let $\varphi: e M \rightarrow B$ be an isomorphism. Then $B=b M$ with $b=s e$ for some $s \in S$, and $\operatorname{ker}(e)=\operatorname{ker}(b)$. Thus, $e \in l_{S}(\operatorname{ker}(e))=l_{S}(\operatorname{ker}(b))=S b \oplus X_{b}$ as $M_{R}$ is AQPinjective, where $X_{b}$ is a left $S$-module. Then $e=t b+x$ with $t \in S$ and $x \in X_{b}$. Hence we have $b=b e=b t b+b x$, and thus $b=b t b$. Let $f=b t$. Then $f^{2}=f$ and $b M=f M$.

Corollary 3.5. Let $M$ be a quasiprojective right $R$-module and let $S=\operatorname{End}\left(M_{R}\right)$. Then $S$ is regular if and only if $M_{R}$ is AQP-injective and im(s) are $M$-projective for every $s \in S$.

Proof. By combining Theorems 3.2, 3.4, and [9, Theorem 37.7], one can complete the proof.

Recall that a ring $R$ is called right P.P. if every principally right ideal of $R$ is projective.

COROLlany 3.6. A ring $R$ is regular if and only if $R$ is right P.P. and right AP-injective. 


\section{Generalizations of principally quasi-injective modules}

Following [3], a module $M$ is said to be weakly injective if, for any finitely generated submodule $N \subseteq E(M)$, we have $N \subseteq X \cong M$ for some $X \subseteq E(M)$.

Corollary 3.7. Let $M_{R}$ be an f.g. module. If $M$ is weakly injective and AQP-injective, then $M$ is injective. In particular, if $R$ is a right $\mathrm{AP}$-injective and a right weakly injective ring, then $R$ is right self-injective.

Proof. Let $x \in E(M)$. Then there exists $X \subseteq E(M)$ such that $M+x R \subseteq X \cong M$, hence $X$ is AQP-injective, and so $M \mid X$ by Theorem 3.4. This shows that $M=X$, so $x \in M$.

We let $S=\operatorname{End}\left(M_{R}\right)$. Following [7], an element $u \in S$ is called a right uniform element of $S$ if $u \neq 0$ and $u(M)$ is a uniform submodule of $M$. In the following, we generalize some results on maximal left ideals of the endomorphism rings of quasiprincipally injective modules and on maximal right ideals of right $\mathrm{AP}$-injective rings to maximal left ideals of the endomorphism rings of AQP-injective modules.

Lemma 3.8. Let $M_{R}$ be a module with $S=\operatorname{End}\left(M_{R}\right)$. Given a set $\left\{X_{s} \mid s \in S\right\}$ of left ideals of $S$, the following are equivalent.

(1) $l_{S}(\operatorname{ker}(s))=S s \bigoplus X_{s}$ for all $s \in S$.

(2) $l_{S}(t M \cap \operatorname{ker}(s))=\left(X_{s t}: t\right)_{l}+S s$ and $\left(X_{s t}: t\right)_{l} \cap S s \subseteq l_{S}(t)$ for all $s, t \in S$, where $\left(X_{s t}\right.$ : $t)_{l}=\left\{x \in S \mid x t \in X_{s t}\right\}$.

Proof. (1) $\Rightarrow(2)$. Let $x \in l_{S}(t M \bigcap \operatorname{ker}(s))$. Then $\operatorname{ker}(s t) \subseteq \operatorname{ker}(x t)$ and so $x t \in l_{S}(\operatorname{ker}(x t)) \subseteq$ $l_{S}(\operatorname{ker}(s t))=S(s t) \bigoplus X_{s t}$. Write $x t=s_{1}(s t)+y$, where $s_{1} \in S$ and $y \in X_{s t}$, then $\left(x-s_{1} s\right) t=$ $y \in X_{s t}$ and hence $x-s_{1} s \in\left(X_{s t}: t\right)_{l}$. It follows that $x \in\left(X_{s t}: t\right)_{l}+S s$. Obviously, $S s \subseteq$ $l_{S}(t M \cap \operatorname{ker}(s))$. If $z \in\left(X_{s t}: t\right)_{l}$, then $z t \in X_{s t} \subseteq l_{S}(\operatorname{ker}(s t))$. Let $t m \in t M \cap \operatorname{ker}(s)$, then $s t m$ $=0$, hence $z t m=0$. This shows that $z \in l_{S}(t M \cap \operatorname{ker}(s))$. Therefore, $l_{S}(t M \bigcap \operatorname{ker}(s))=$ $\left(X_{s t}: t\right)_{l}+S s$. If $s^{\prime} \in\left(X_{s t}: t\right)_{l} \cap S s$, then $s^{\prime} s t \in X_{s t} \cap S(s t)=0$, and thus $s^{\prime} s \in l_{S}(t)$.

(2) $\Rightarrow(1)$. Let $t=1$.

Lemma 3.9. Let $M_{R}$ be an AQP-injective module with $S=\operatorname{End}\left(M_{R}\right)$ and an index set $\left\{X_{s} \mid\right.$ $s \in S\}$ of ideals such that $X_{s t}=X_{t s}$ for all $s, t \in S$. If $0 \neq u(M)$ is a uniform submodule of $M$, define $M_{u}=\{s \in S \mid \operatorname{ker}(s) \cap u(M) \neq 0\}$. Then $M_{u}$ is the unique maximal left ideal of $S$ which contains $\sum_{s \in S}\left(X_{s u}: u\right)_{l}$.

Proof. It is easy to see that $M_{u}$ is a left ideal. Let $t \in\left(X_{s u}: u\right)_{l}$, then $t u \in X_{s u}$, and thus $t u s \in$ $X_{s u} \cap S(u s)=X_{u s} \cap S(u s)$, since $X_{s u}=X_{u s}$ is an ideal. Then $t u s=0$ and so $t \in M_{u}$ if $u s \neq 0$. If $u s=0$, then $l_{S}(\operatorname{ker}(u s))=0$, and so $X_{s u}=X_{u s}=0$. This shows that $t u=0$ and hence $t \in M_{u}$. Consequently, $\left(X_{s u}: u\right)_{l} \subseteq M_{u}$ for all $s \in S$. Now if $s \notin M_{u}$, then $\operatorname{ker}(s) \bigcap u M=0$, and so $S=\left(X_{s u}: u\right)_{l}+S s$ by Lemma 3.8, hence $S=M_{u}+S s$, showing that $M_{u}$ is a maximal left ideal.

Finally, let $L$ be a left ideal of $S$ such that $\sum_{s \in S}\left(X_{s u}: u\right)_{l} \subseteq L \neq M_{u}$. Then, as above, $S=\left(X_{s u}: u\right)_{l}+S s$ for any $s \in L-M_{u}$. Therefore, $L=S$.

Lemma 3.10. Let $M_{R}$ be AQP-injective with $S=\operatorname{End}\left(M_{R}\right)$ and an index set $\left\{X_{s} \mid s \in S\right\}$ of ideals such that $X_{s t}=X_{t s}$ for all $s, t \in S$ and let $W=u_{1} M \oplus u_{2} M \oplus \cdots \oplus u_{n} M$ be a direct sum of uniform submodules $u_{i} M$ of $M$, where each $u_{i} \in S$. If $T \subseteq S$ is a maximal left ideal 
not of the form $M_{u}$ for any $u \in S$ such that $u M$ is uniform, then there is $t \in T$ such that $\operatorname{ker}(1-t) \cap W$ is essential in $W$.

Proof. Since $T \neq M_{u_{1}}$, let $\operatorname{ker}(a) \bigcap u_{1} M=0, a \in T$, then $\operatorname{ker}\left(a u_{1}\right) \subseteq \operatorname{ker}\left(u_{1}\right)$, and so $u_{1} \in$ $l_{S}\left(\operatorname{ker}\left(a u_{1}\right)\right)=S\left(a u_{1}\right) \oplus X_{a u_{1}}$. Thus, there exists $s \in S$ such that $(1-s a) u_{1} \in X_{a u}$, and so $1-s a \in\left(X_{a u_{1}}: u_{1}\right)_{l} \subseteq M_{u_{1}}$. Let $a_{1}=s a$. If $1-a_{1} \in M_{u_{i}}$ for all $i$, we are done. If, say, $1-a_{1} \notin M_{2}$, then $\left(1-a_{1}\right) u_{2} M$ is uniform (being isomorphic to $u_{2} M$ ), so, as above, $\left(1-a^{\prime}\right) \in M_{\left(1-a_{1}\right) u_{2}}$ for some $a^{\prime} \in T$. Let $a_{2}=a^{\prime} t a_{1}-a^{\prime} a_{1}$, then $1-a_{2} \in M_{u_{1}} \cap M_{u_{2}}$, continue in this way to obtain $t \in S$, such that $\operatorname{ker}(1-t) \bigcap u_{i} M \neq 0$ for each $i$, Lemma 3.10 follows.

Theorem 3.11. Let $M_{R}$ be a self-generator with finite Goldie dimension and $S=\operatorname{End}\left(M_{R}\right)$. If $M_{R}$ is AQP-injective with an index set $\left\{X_{s} \mid s \in S\right\}$ of left ideals of $S$ such that $X_{s t}=X_{t s}$ for all $s, t \in S$, then

(1) if $T$ is a maximal left ideal of $S$, then $T=M_{u}$ for some $u \in S$ such that $u M$ is a uniform submodule of $M$,

(2) $S / J(S)$ is semisimple.

Proof. Since $M$ is a self-generator, every uniform submodule of $M$ contains an $M$-cyclic submodule. Therefore, we can assume that $W=u_{1} M \oplus u_{2} M \oplus \cdots \bigoplus u_{n} M$ is essential as $M_{R}$ has finite Goldie dimension. If $T$ is not of the form $A_{u}$ for some right uniform element of $u \in S$, then by Lemma 3.10, there exists some $t \in T$ such that $\operatorname{ker}(1-t) \cap W$ is essential in $W$, so $\operatorname{ker}(1-t)$ is essential in $M$. By Theorem 3.3, $1-t \in J(S) \subseteq T$, a contradiction. This proves (1). As to (2), if $s \in M_{u_{1}} \cap \cdots \cap M_{u_{n}}$, then $\operatorname{ker}(s) \cap u_{i} M \neq 0$ for each $i$, whence $\operatorname{ker}(s)$ is essential in $M$. Hence, $s \in J(S)$, proving (2).

\section{Acknowledgment}

The authors are grateful to the referee for useful suggestions and remarks.

\section{References}

[1] F. W. Anderson and K. R. Fuller, Rings and Categories of Modules, Springer, New York, 1974.

[2] N. Ding, M. F. Yousif, and Y. Zhou, Modules with annihilator conditions, Comm. Algebra 30 (2002), no. 5, 2309-2320.

[3] S. K. Jain and S. R. López-Permouth, Rings whose cyclics are essentially embeddable in projective modules, J. Algebra 128 (1990), no. 1, 257-269.

[4] W. K. Nicholson, J. K. Park, and M. F. Yousif, Principally quasi-injective modules, Comm. Algebra 27 (1999), no. 4, 1683-1693.

[5] W. K. Nicholson and M. F. Yousif, Principally injective rings, J. Algebra 174 (1995), no. 1, 7793.

[6] S. S. Page and Y. Zhou, Generalizations of principally injective rings, J. Algebra 206 (1998), no. 2, 706-721.

[7] N. V. Sanh and K. P. Shum, Endomorphism rings of quasi-principally injective modules, Comm. Algebra 29 (2001), no. 4, 1437-1443.

[8] N. V. Sanh, K. P. Shum, S. Dhompongsa, and S. Wongwai, On quasi-principally injective modules, Algebra Colloq. 6 (1999), no. 3, 269-276. 
1860 Generalizations of principally quasi-injective modules

[9] R. Wisbauer, Foundations of Module and Ring Theory, Algebra, Logic and Applications, vol. 3, Gordon and Breach Science, Pennsylvania, 1991.

Zhu Zhanmin: Department of Mathematics, Jiaxing University, Jiaxing, Zhejiang 314001, China E-mail address: zhanmin_zhu@hotmail.com

Xia Zhangsheng: Department of Mathematics, Hubei Institute for Nationalities, Enshi, Hubei 445000, China

E-mail address: xzsw8577@163.com

Tan Zhisong: Department of Mathematics, Hubei Institute for Nationalities, Enshi, Hubei 445000, China

E-mail address: es_tzs@hotmail.com 


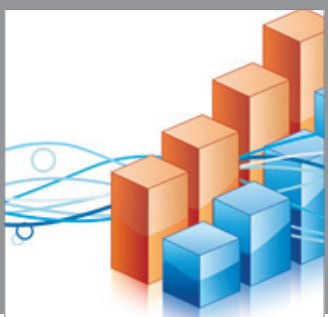

Advances in

Operations Research

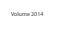

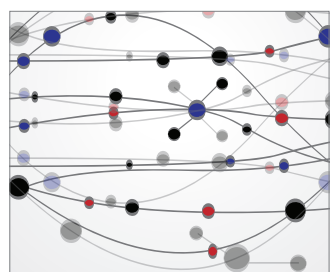

\section{The Scientific} World Journal
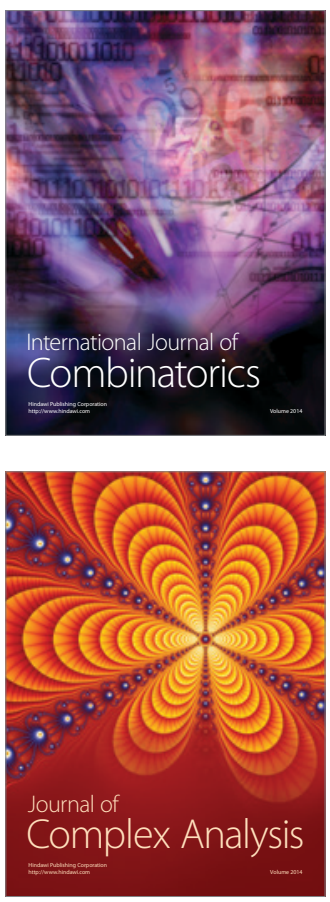

International Journal of

Mathematics and

Mathematical

Sciences
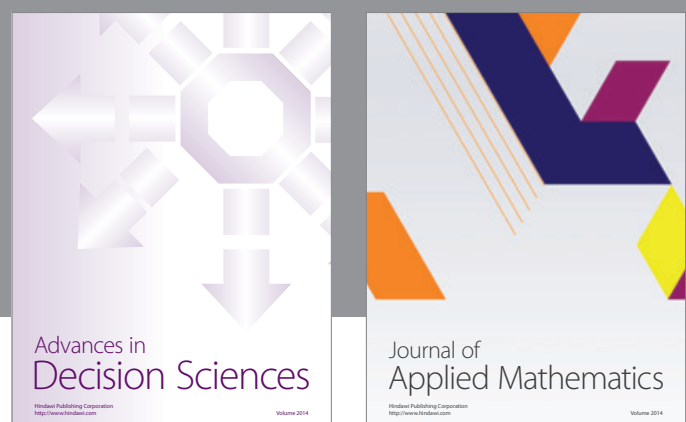

Journal of

Applied Mathematics
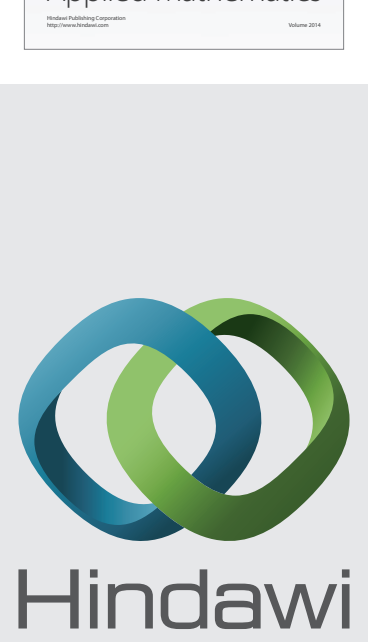

Submit your manuscripts at http://www.hindawi.com
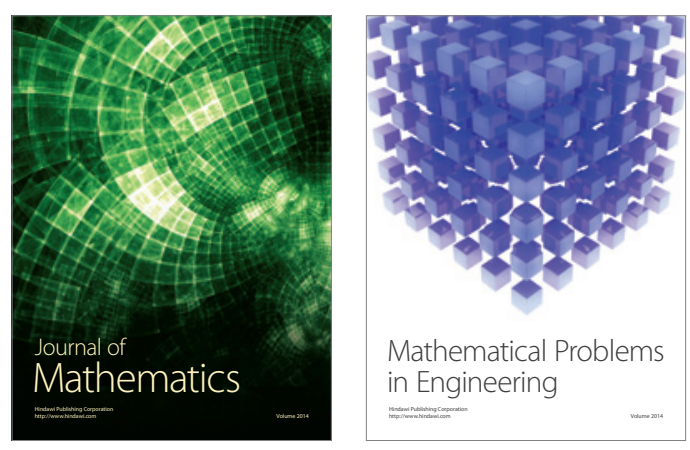

Mathematical Problems in Engineering
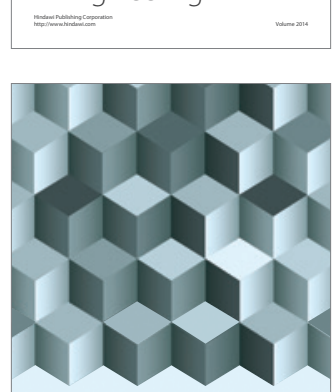

Journal of

Function Spaces
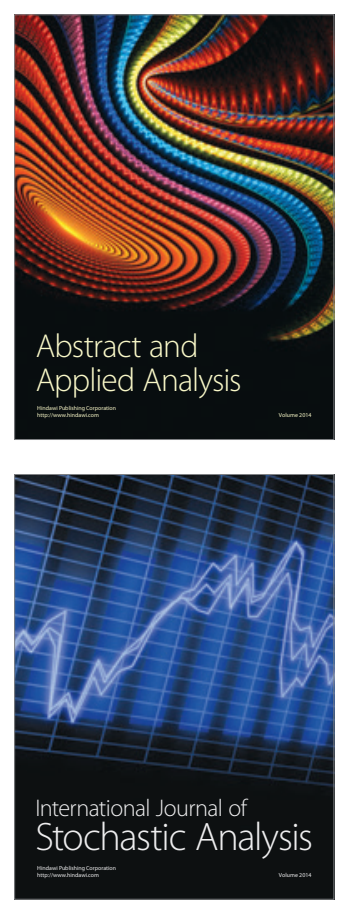

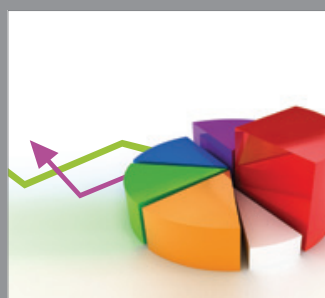

ournal of

Probability and Statistics

Promensencen
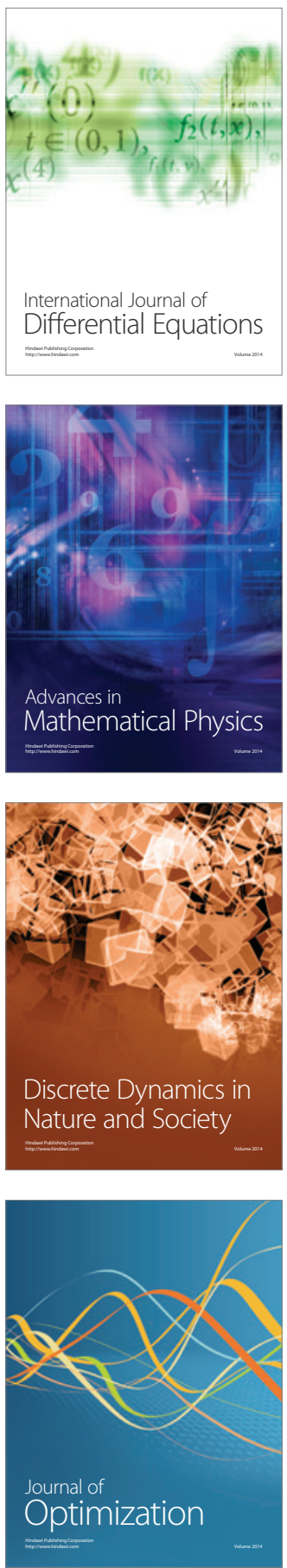